\title{
Clinical outcome of preimplantation genetic diagnosis for cystic fibrosis: the Brussels' experience
}

\author{
Kathelijn Keymolen ${ }^{*},{ }^{1}$, Veerle Goossens ${ }^{1}$, Martine De rycke ${ }^{1}$, Karen Sermon ${ }^{1}$, \\ Kristel Boelaert ${ }^{1}$, Maryse Bonduelle ${ }^{1}$, André Van Steirteghem ${ }^{2}$ and Inge Liebaers ${ }^{1}$
}

${ }^{1}$ Centre for Medical Genetics, Vrije Universiteit Brussel, Belgium; ${ }^{2}$ Centre for Reproductive Medicine, Vrije Universiteit Brussel, Belgium

Preimplantation genetic diagnosis is an alternative for prenatal diagnosis that makes it possible to perform the diagnosis of a chromosomal or monogenic disorder at the preimplantation embryo level. Cystic fibrosis is one of the monogenic diseases for which PGD can be performed. In this study, we looked at the requests and PGD cycles for this particular disorder over an 11-year period. Sixty-eight percent of the requests eventually led to at least one complete PGD cycle. In $80 \%$ of the cycles, an embryo transfer was performed and an ongoing pregnancy was obtained in $22.2 \%$ of the cycles with oocyte retrieval. After embryo transfer, a couple had $27.8 \%$ chance of giving birth to a liveborn child. No misdiagnosis was recorded. The rate of perinatal deaths/stillborn children was relatively high, but no excess of major congenital anomalies was observed in the surviving children.

European Journal of Human Genetics (2007) 15, 752-758; doi:10.1038/sj.ejhg.5201834; published online 18 April 2007

Keywords: preimplantation genetic diagnosis; pgd; cystic fibrosis; cf; children

\section{Introduction}

Cystic fibrosis (CF) (OMIM 219700) is an autosomal recessive disorder affecting the respiratory and gastrointestinal tract and causing fertility problems in males. Better treatment options seriously improve life expectancy of CF patients; however, it remains a severe disease with a great impact on quality of life, which parents may want to avoid in their offspring.

Preimplantation genetic diagnosis (PGD) is an alternative for prenatal diagnosis, giving couples with a high genetic risk the opportunity to have unaffected children without having to consider termination of pregnancy. ${ }^{1}$

The technique was performed for the first time in 1990 and aimed at the selection of female embryos for X-linked disorders. ${ }^{1}$ Worldwide CF was the first autosomal mono-

*Correspondence: Dr K Keymolen, Centre for Medical Genetics, University Hospital, Vrije Universiteit Brussels, Laarbeeklaan 101, 1090 Brussels, Belgium.

Tel: + 3224 776071; Fax: + 3224 776860;

E-mail: Igenknk@az.vub.ac.be

Received 26 June 2006; revised 13 March 2007; accepted 17 March 2007; published online 18 April 2007 genic disorder for which a specific PGD could be offered. ${ }^{2}$ Single-cell analysis of p.F508del, a common CF mutation, was also the first clinical PGD application at our centres. ${ }^{3}$ Since its introduction, diagnostic approaches have been adapted to be able to help carriers of different CF mutations and reduce the risk of contamination and allele drop-out (ie the failure of amplification of one of two alleles in a heterozygous cell) rates, two characteristics of single-cell polymerase chain reaction (PCR). ${ }^{4,5,6,7}$

The figures for the success rate that are used in the counselling of couples are derived from a cohort of PGD cycles for various disorders with different modes of inheritance. Yet, it is not unthinkable that these figures could differ for a particular disease, and this study aims at giving specific data on the outcome of PGD for CF. To the best of our knowledge, this is one of the first studies to look at disease-specific PGD outcome.

\section{Materials and methods}

Over a period of 11 years, from January 1992 to December 2002, couples who contacted the Centre for Medical 
Genetics with a request for information concerning PGD for $\mathrm{CF}$ were registered and data regarding the country of origin, assessment for $\mathrm{CF}$, maternal age, reason for opting for PGD and mutations involved were recorded. Of the 69 included couples, 7 were never seen at our clinic, but only corresponded with the medical staff of the Centre by mail or e-mail, and 15 couples did not have PGD.

Before the start of a PGD cycle, the couples were informed about the procedure by a clinical geneticist, a psychologist, a fertility gynaecologist and a fertility nurse. All the couples signed an informed consent in which the general principles of PGD were summarised and in which the pregnancy rate per cycle $(20-25 \%)$ and the risk of misdiagnosis $(1-5 \%)$ were specified. The couples also agreed that affected embryos and embryos not suitable for transfer or freezing could be used for research.

For each PGD cycle, we collected information about the number of oocytes retrieved, the number of oocytes that were inseminated using intracytoplasmic sperm injection (ICSI), the number of embryos for biopsy, the occurrence of embryo transfer, pregnancy, prenatal or postnatal confirmation of PGD, birth data and follow-up of the children.

All PGD couples underwent in vitro fertilization (IVF) treatment. After controlled ovarian stimulation combining GnRH agonist or antagonists, urinary or recombinant gonadotrophins and hCG, ultrasound-guided transvaginal oocyte retrieval was carried out. ${ }^{8}$ In all but two cycles, ICSI was performed as described by Devroey and Van Steirteghem. ${ }^{9}$ After ICSI, fertilization and embryo development were assessed daily. Embryos with at least six cells on day 3 were biopsied. One or two blastomeres were aspirated through a hole in the zona pellucida. ${ }^{10}$ Until May 1998, one blastomere was removed from embryos with less than seven cells and two were taken from embryos with at least seven cells. After May 1998, always two blastomeres were biopsied. Except for the first four cycles, where cells were transferred to $\mathrm{H}_{2} \mathrm{O}$ and incubated at $95^{\circ} \mathrm{C}$, cell lysis was obtained by incubation for $10 \mathrm{~min}$ at $65^{\circ} \mathrm{C}$ in an alkaline lysis buffer, which was later neutralized with neutralisation buffer. PCR-based procedures were performed as described by Goossens et $\mathrm{al}^{4,5}$ on the biopsied blastomeres. In the beginning, conventional PCR was used with two consecutive rounds of PCR with nested or hemi-nested primers. Later, fluorescent PCR was performed with primers labelled with fluorescein when using the automated laser fluorescence (ALF, Amersham Pharmacia Biotech, The Netherlands) or with Cy5 when using the ALF express. Direct mutation analysis was performed for p.F508del, p.N1303 K, p.1717-1G $>$ A and p.2789 + 5G $>$ A mutations. PGD based on linked markers used intragenic (M470V, IVS8CA, IVS17BTA) and extragenic (D7S486, D7S490, D7S480) polymorphisms, which could be combined by duplex PCR. Genetically non-affected (heterozygous carrier or homozygous non-carrier) and morphologically good quality embryos were transferred to the uterus on the fourth or fifth day. ${ }^{11}$ Only when the results in the two blastomeres of an embryo were concordantly non-affected, the embryo was suitable for transfer. After transfer, blood sampling for hCG (at 12 days) and ultrasound examinations (at 6 weeks) were performed to document a pregnancy. ${ }^{12}$ Prenatal diagnosis was offered because of the small but existing risk of misdiagnosis and/or because of maternal age. The possibility of performing a fetal karyotype, besides the DNA analysis for CF, was discussed with the couples, and all those opting for prenatal diagnosis, agreed with the chromosomal analysis.

Questionnaires about the evolution of the pregnancy were sent to the future parents and their obstetricians. After birth, parents and children were invited to the outpatient clinic of the Centre for Medical Genetics to review the child's medical history and for a detailed physical examination. The presence of major malformations (defined as an anomaly that causes functional impairment or requires surgical correction) was recorded. When it was not possible for the family to go to the hospital, questionnaires were sent to be filled in by the parents or the child's paediatrician. A follow-up was foreseen at 2 months, at 1 year and at 2 years as described by Bonduelle et al. ${ }^{13}$

\section{Results}

A total of 69 couples contacted the Centre and requested information about PGD. Twenty-five couples (36.2\%) came from Belgium, 13 from Germany $(18.8 \%), 8$ from Israel (11.6\%), 6 from Italy (8.7\%), 5 from France (7.2\%) and a few couples from the UK, USA, The Netherlands, Canada, India, Luxemburg and Switzerland.

The way their CF risk was assessed and the reasons for choosing PGD are shown in Tables 1 and 2.

In the 69 couples, the p.F508del mutation was the most frequent mutation followed by p.W1282X, p.N1303K, p.G542X, p.R117H, 5T variant and a variety of other less frequent mutations.

\section{Couples without PGD cycle}

The reason why a (complete) PGD cycle could not be performed could only be ascertained for nine couples. Three couples achieved one spontaneous pregnancy and one couple had two spontaneous pregnancies. Prenatal diagnosis was performed during these five pregnancies, revealing four heterozygous carrier foetuses and one affected foetus.

One couple started ovarian stimulation, but the cycle was cancelled because of low ovarian response. One couple wanted not only PGD for CF, but also sex selection for nonmedical reasons, which is not performed at our centre. For the couple for which there was also a risk of conceiving a child affected with Tay-Sachs, no combined PGD could be offered, and for another couple the CFTR mutations were 
Table 1 Assessment of CF risk

\begin{tabular}{lccc}
\hline & $\begin{array}{c}\text { Couples } \\
\text { with PGD } \\
(\mathrm{n}=47)\end{array}$ & $\begin{array}{c}\text { Couples } \\
\text { without PGD } \\
(\mathrm{n}=22)\end{array}$ & $\begin{array}{c}\text { All couples } \\
(\mathrm{n}=69)(\%)\end{array}$ \\
\hline $\begin{array}{l}\text { CF risk assessment } \\
\text { Affected child or foetus }\end{array}$ & 23 & 14 & $37(53.6)$ \\
$\begin{array}{l}\text { CBAVD (without other } \\
\text { CF complaints) } \\
\quad \text { During fertility work-up }\end{array}$ & 7 & 3 & $10(14.5)$ \\
$\begin{array}{l}\text { (not CBAVD) } \\
\text { Positive family history }\end{array}$ & 3 & & $10(14.5)$ \\
$\begin{array}{l}\text { CF patient (with } \\
\begin{array}{c}\text { CBAVD in males) } \\
\text { Unknown }\end{array}\end{array}$ & 4 & 2 & $5(7.2)$ \\
$\begin{array}{l}\text { Preconceptual } \\
\text { screening }\end{array}$ & & 2 & $4(5.8)$ \\
\hline
\end{tabular}

Table 2 Reasons for choosing PGD

\begin{tabular}{lccc}
\hline & $\begin{array}{c}\text { Couples } \\
\text { with PGD } \\
(\mathrm{n}=47)\end{array}$ & $\begin{array}{c}\text { Couples } \\
\text { without PGD } \\
(\mathrm{n}=22)\end{array}$ & $\begin{array}{c}\text { All couples } \\
(\mathrm{n}=69)\end{array}$ \\
\hline $\begin{array}{l}\text { Reason for choosing/informing about PGD } \\
\quad \text { Fertility problems }\end{array}$ & 24 & 7 & $31(44.9 \%)$ \\
$\begin{array}{l}\text { Objection to abortion } \\
\text { History of termination }\end{array}$ & 15 & 2 & $17(24.6 \%)$ \\
of pregnancy & 8 & 1 & $9(13 \%)$ \\
$\quad$ Unknown & & 11 & $11(15.9 \%)$ \\
Other & & 1 & $1(1.4 \%)$ \\
\hline
\end{tabular}

not known at the time of their request. One couple divorced and so had no child wish anymore.

\section{Couples with PGD cycle(s)}

PGD was performed for 47 couples and CF remained thus by far the most frequent monogenic disease for which PGD was performed in our centres. It takes more than half (55\%) of all PGD cycles for autosomal recessive disorders on its account.

The 47 couples were living in 12 different countries (Belgium: 14; Germany: 10; Israel: 7; France: 4; UK: 3; Italy: 3; Canada: 1; India: 1; Luxemburg: 1; The Netherlands: 1; Switzerland: 1 ; USA: 1 ). Their way of assessing the CF risk and the reasons for choosing PGD are summarised in Tables 1 and 2 . The CFTR genotypes of the couples are shown in Table 3.

\section{PGD cycles and outcome}

Each couple underwent an average of 1.9 cycles (range $1-6)$.

In the 90 cycles that were performed, 1127 cumulusoocyte complexes (COC) were retrieved, and ICSI was performed in 921 metaphase II oocytes (81.7\%). A total of 461 embryos (50\%) could be biopsied. In 80 cycles, all
Table 3 Genotypes of the couples with PGD cycles

\begin{tabular}{|c|c|c|}
\hline Female partner & Male partner & $\begin{array}{c}\text { Number of couples with } \\
\text { this genotype }\end{array}$ \\
\hline p.F508del/- & p.F508del/- & 17 \\
\hline p.F508del/- & p.R117H/- (7T/9T) & 1 \\
\hline p. $2789+5 G>A /-$ & p.D110H/p.D110H & 1 \\
\hline p.G542X/- & p.F508del/- & 1 \\
\hline p.R334Q/- & p.F508del/- & 1 \\
\hline p.R553X/- & p.F508del/- & 2 \\
\hline p.1717-1G >A & p. $2183 \mathrm{AA}>\mathrm{G} / 5 \mathrm{~T}$ & 1 \\
\hline p.F508del/- & p.F508del/? & 2 \\
\hline p.1303K/- & p.G542X/p.R117H & 1 \\
\hline p.F508del/- & $5 \mathrm{~T} / ?$ & 1 \\
\hline p.F508del/- & p.G194T/5T & 1 \\
\hline $\begin{array}{l}\text { p.F508del/ } \\
\text { p.3272-26A >G }\end{array}$ & p.R1162X/- & 1 \\
\hline p.F508del/- & $\begin{array}{l}\text { p.F508del/ } \\
\text { p.R117H (7T/9T) }\end{array}$ & 1 \\
\hline p.F508del/- & $\begin{array}{l}\text { p.F508del/ } \\
\text { p.F508del }\end{array}$ & 1 \\
\hline p.R334W/- & $\begin{array}{l}\text { p.F508del/ } \\
\text { p.F508del }\end{array}$ & 1 \\
\hline p.F508del/- & $\begin{array}{l}\text { p.F508del/ } \\
\text { p.M265R }\end{array}$ & 1 \\
\hline p.F508del/- & $? / ?$ & 2 \\
\hline p.F508del/- & p.N1303K/- & 1 \\
\hline p.Q493X/- & p.F508del/- & 1 \\
\hline p.F508del/- & p.R1162X/- & 1 \\
\hline p.4218insT/- & p.N1303K/- & 1 \\
\hline p.G673X/- & p.F508del/- & 1 \\
\hline p.W1282X/- & p.G542X/- & 1 \\
\hline p.F508del/- & p.W1282X/- & 1 \\
\hline p.W1282X/- & p.F508del/- & 2 \\
\hline p.F508del/- & p.G551D/- & 1 \\
\hline p.D1168G/- & p.L206W/- & 1 \\
\hline
\end{tabular}

biopsied embryos had two blastomeres removed. In two cycles only one cell could be taken from the available embryos, and in five cycles there was a mixture of one and two blastomeres per embryo taken. For three cycles this information was not available.

For 24 couples direct mutation detection was used, for 8 couples a combination of polymorphisms was performed and for 14 couples a duplex PCR for a mutation and a polymorphism was chosen. For one couple, two different strategies were used in subsequent cycles (first mutation detection and later a duplex PCR for a mutation and a linked marker). When one blastomere was available and only the non-mutant haplotype at the CF locus was identified, the embryo was suitable for transfer, but when two blastomeres were biopsied and both showed only one haplotype, the embryo was not suitable for transfer. The rates of allele drop out and their evolution over time are under study and will be published by our group in the near future. In 72 cycles (80\%), a transfer (ET) of 1-3 embryos was performed.

After fresh embryo transfer, positive serum hCG was found in 23 women, leading to 27 foetal sacs and 25 positive foetal heart beats (15 singleton and 5 twin pregnancies). As a result, 25 children were liveborn. 
If we express these results per cycle with oocyte retrieval, this means that in each cycle there was an average of 12.5 COCs, giving 5.1 embryos to be biopsied with an $80 \%$ chance of having an embryo transfer and a $22.2 \%$ chance of having an ongoing pregnancy with the delivery of a child.

Once a couple had reached the stage of an embryo transfer, they had $31.9 \%$ chance of having a positive hCG and a $27.8 \%$ chance of giving birth to a liveborn child.

There was no statistically significant difference between the outcome results for couples with $(n=24)$ and without $(n=23)$ a fertility problem (Fisher's exact test). The mean maternal age in the group with a child was 31.14 years, whereas it was 31.96 years in the group without a child.

In 17 cycles (19\%), one or more embryos could be cryopreserved. Subsequently, six couples had one cycle and one couple had two cycles with transfer of frozen/thawed embryos. No pregnancy was obtained but since the small sample size and a pregnancy rate of about 5\% for cryopreserved embryos without biopsy, this was not surprising.

\section{Follow-up of the children}

Of the 25 liveborn children, 14 were boys and 11 girls. Fifteen children were singletons and 10 children were one of a twin. Prenatal diagnosis was performed for 13 children (11 chorionic villus samplings and 2 amniocenteses), confirming the PGD DNA result for CF, and showing a normal karyotype. For one child, a postnatal DNA test for CF was performed, and in two children a sweat test was available, which produced normal results. For the remaining nine children, the follow-up data did not reveal any clinical signs of CF.

Three deliveries were premature (32 weeks). It concerned two singleton pregnancies and one twin. During a twin pregnancy, chylothorax was observed in one of the foetuses and intrauterine drainage was carried out. Unfortunately, there was recurrence of the chylothorax postnatally, and the boy died on the second day after birth. No malformations were identified through necropsy and the underlying mechanism for chylothorax therefore remains unknown.

Of the remaining 24 children, follow-up data were available for 21 of them at 2 months of age. Birth weights were known for 23 children and ranged from 2700 to $4010 \mathrm{~g}$ (mean $3473 \mathrm{~g}$ ) for term-born singleton boys; from 3100 to $3640 \mathrm{~g}$ (mean $3433 \mathrm{~g}$ ) for term-born singleton girls, from 2800 to $3500 \mathrm{~g}$ (mean $3083 \mathrm{~g}$ ) for term-born boys from twins and from 2295 to $2500 \mathrm{~g}$ (mean $2376 \mathrm{~g}$ ) for term-born girls from twins. For the four prematurely born children, only one boy was small for gestational age (1400 g at 32 weeks). No major malformations were observed in these children. It should be noted that 10 out of the 21 children were born in/after 2000, so that they had not yet reached the age of 12 or 24 months at the time of the study.
For 11 children follow-up data were available at 12 months, and for 10 children at 24 months. These data revealed heart murmur in one, capillary haemangioma in two, congenital hip subluxation in two, congenital junctional nevus in one and gastro-oesophageal reflux in three children. In one child fine, motor delay was observed at 1 year 11 months, and in one girl there was an important psychomotor retardation.

\section{Follow-up of the PGD couples}

In the group of 47 PGD couples, follow-up information after December 2002 is available for 14 couples. Twelve of them continued PGD. For two couples, this led to a pregnancy, but three others turned to the use of donor sperm, after at least one other PGD cycle had been performed without success (sperm donors were screened for the 29 most frequent CFTR mutations). In two of the latter couples, a pregnancy occurred. One couple became pregnant spontaneously after having tried another PGD treatment, and one couple stopped PGD and immediately became pregnant. These couples performed prenatal diagnosis for $\mathrm{CF}$ and the foetuses were shown not to be affected. One couple had intrauterine inseminations with prenatal diagnosis in case of pregnancy, and another couple had transfer of frozen embryos.

\section{Discussion}

Couples interested in PGD should be carefully counselled about all practical modalities of the treatment and about their chances of taking a healthy baby home. ${ }^{12,14}$ The present study provides disease-specific data both about the characteristics of the couples requesting/undergoing PGD and about the outcome of the PGD cycles. This approach should enable counsellors to give the couples more specific information about and more accurate assessments of their chances of taking a baby home after PGD treatment.

As far as the characteristics of the couples are concerned, most of those requesting PGD were Belgians, but only $14 / 25$ went through with the procedure after being counselled. Most of the requests from abroad came from Germany and Israel, and of these respectively 10/13 and 7/8 went through with PGD. In all probability, the foreign couples who requested PGD were well informed about the procedure before they contacted our centre and they mainly turned to our centre because of the availability of the procedure and sometimes because PGD was forbidden in their own country.

Compared to other monogenic diseases for which PGD is offered, a relatively high proportion (24/47) of the CF-PGD couples chose PGD because of existing fertility problems (Table 2). This marked increase (51\% versus approximately one-third in other PGD cycles) ${ }^{12,15,25}$ was due to several factors. 
First, male infertility is one of the clinical manifestations of CF because of CBAVD. The latter can be either the only symptom, or it may be part of the 'fullblown' CF clinical presentation. Secondly, screening for the most frequent CFTR mutations is often offered to couples treated in fertility clinics (for reasons other than CBAVD). ${ }^{16,17}$ In some of the cases of CBAVD, the male partner was carrier of the $5 \mathrm{~T}$ allele, which is a variant in the polythymidine tract of intron 8 regulating the skipping of exon $9 .{ }^{18}$ In these couples, embryos carrying one classic CFTR mutation and a 5T allele were not transferred. However, all these couples were also at risk for having embryos with two classic CFTR mutations and they needed ICSI because of the CBAVD, so that the primary reason for doing PGD was never exclusively avoiding a child with classic mutation/5T. During the study period, we had no requests from nonCBAVD couples in whom one partner was a $5 \mathrm{~T}$ carrier and one carrier of a classic mutation. However, recently several such couples were referred to our centre. After discussion of the risks of having a boy with CBAVD and the related reproductive options as well as the possibility of mild CF-like symptoms, these couples choose to perform neither prenatal nor preimplantation genetic diagnosis. It is our feeling that couples may want medical assistance in order to avoid a severely affected child, but that they are more reluctant to this when it concerns milder symptomatology. Nevertheless each individual request should be carefully evaluated. The TG status was not analysed for the couples in this study but with accumulating evidence that this polymorphism affects the penetrance of the $5 \mathrm{~T}$ allele, this should certainly be considered in future couples.

With the increasing life expectancy of CF patients, parenthood will become a more frequently discussed issue and counselling should be offered to all of them. As for couples with a one in four risk, PGD can also be proposed to couples with a one in two risk of having a CF child. Especially in male CF patients, PGD could seem to be a logical option since they always need medically assisted reproductive techniques. However, despite the technical feasibility of PGD in these couples, we should remember that it is important to carefully investigate the patient's health before starting a PGD cycle. Women with CF should not be accepted in a PGD programme unless a detailed report from their treating physician is available, stating that there are no contraindications for a pregnancy. It is also advisable that these couples are seen by a psychologist and that the partner's knowledge regarding CF is ascertained. Issues such as becoming a single parent after the CF patient's death should be addressed. ${ }^{19}$

Twenty-three different CFTR mutations were observed in our CF-PGD couples. As expected, p.F508del was the most prominent. Developing a specific PCR procedure for each mutation would be very time consuming and very expensive. Therefore, the use of linked extragenic and intragenic markers was introduced, thereby increasing the number of couples to whom PGD can be offered. ${ }^{4,5}$ By using these markers, allele drop out and contamination by other DNA sources can also be detected, and this decreases the risk of misdiagnosis or loss of healthy embryos. ${ }^{4-7}$

Although the number of couples included in this study was still small and follow-up data were incomplete, we think that this study demonstrated that the outcome of PGD for CF was comparable to the latest data from the ESHRE PGD consortium. ${ }^{25}$ Data collection $\mathrm{V}$ from this consortium showed that in the group of PGD for monogenic disorders there was an average of 13 oocytes collected per oocyte retrieval, a positive hCG rate of $35.9 \%$ per embryo transfer and a clinical pregnancy rate of $28 \%$ per embryo transfer, compared to 12.5 oocytes per retrieval, $31.9 \%$ positive HCG per transfer and $27.8 \%$ births of liveborn children per transfer in the present study. For the 90 cycles of PGD for CF performed, the chance of having an ongoing pregnancy was $22.2 \%$ per cycle started. Since only in a minority of the cycles there was exclusively one blastomere per embryo removed, we cannot draw any conclusion on whether one or two blastomere biopsy could influence the outcome of the PGD cycle but nowadays a randomized trial (1 versus 2 blastomeres biopsy) is going on in our centres.

It is noteworthy that $55 \%(11 / 20)$ of the successful pregnancies were obtained at the first PGD cycle and 30\% (6/20) after two cycles. Only two successful pregnancies occurred after three cycles, and one pregnancy after five cycles. Taking into account that one couple had two subsequent pregnancies after PGD, this means that $40.4 \%$ of the couples took at least one baby home after a mean of two cycles. These figures were in line with earlier reported data on the success rate of PGD for monogenic disorders. ${ }^{21,23,25}$

In the study group, the proportion of children born after twin pregnancy was still high (10/25). Although only one twin pregnancy ended prematurely at 32 weeks, the aim for the future should be to reduce the number of multiple pregnancies, since it remains associated with higher mortality and morbidity. The success rate achieved with single embryo transfer ${ }^{24}$ has led to a change in the legal provisions in Belgium since July 2003; only one embryo may now be transferred in the first two IVF trials of women under 36 years. This will give rise to a serious decrease of multiplets.

No major malformations were observed in the 24 surviving children after CF-PGD. In one girl, psychomotor delay without congenital malformations was observed. Despite extensive examinations by her treating physicians, no etiology could be found.

In total, we observed an adverse outcome in 2 of the 25 children ( 1 in 25 perinatal death and 1 in 25 mental retardation). The rate of stillborn children/perinatal deaths for the whole group of children born after PGD or aneuploidy screening in our centres was 20/437 (personal communication Liebaers, Brussels, 2003). The majority of 
these deceased children (16 of the 20) were part of a multiplet, as was also the case for the child in the CF-PGD cohort. We hope the perinatal mortality rate will decrease in the future in line with the falling number of twins.

All available pre- and postnatal data allowed us to state that in this series there were no arguments for misdiagnosis. However, because there still is a small risk of misdiagnosis ${ }^{20,21,25}$ for PGD in general, we continue to inform couples about the possibility and suggest they perform prenatal diagnosis in case of pregnancy after PGD. In this series, only $42.5 \%$ of the pregnant women below 35 years of age underwent chorionic villus biopsy or amniocentesis in contrast to $75 \%$ of the pregnant women above 35 years. This was in contrast with the observation by Bonduelle et $a l^{22}$ who found that in ICSI pregnancies without PGD, younger women were more likely to have a prenatal diagnosis performed than those with maternal age-related risk. In the present study, $63.6 \%$ of the couples performing prenatal diagnosis had fertility problems and $36.4 \%$ had a first affected child. In the group who did not perform prenatal diagnosis, only $33.3 \%$ had infertility and $66.6 \%$ had a child with CF. This difference was statistically not significant ( $p=0.3$, Fisher's exact test) and there was no increased maternal age in the group with fertility problems (mean maternal age 31.4 years versus 31.7 years in the fertile group). Future and larger prospective studies on PGD outcome should certainly also address this issue on prenatal diagnosis.

Concerning the follow-up of the couples after December 2002 , data were available for only 14 of the 47 couples. Twelve of these couples had at least one other PGD cycle. In three of them, it concerned treatment for a second PGD child. With time, 3 of the 12 couples switched to the use of donor sperm. All three of them had fertility problems of male origin (two CBAVD and one cryptorchidism) and maybe this is what finally made it possible for these couples to use donor gametes. One of the couples who first continued PGD then became pregnant spontaneously, and prenatal diagnosis was performed. It was striking that this couple first chose PGD because they had moral objections to abortion. However, after three failed PGD treatments, they changed their minds.

For the three remaining couples, one became spontaneously pregnant and two had plans for other, notspecified, fertility treatments. Financial matters were never evoked in this small sample although this could for some couples also be a limiting factor. For Belgian couples most expenses for a PGD treatment are covered by the health insurance, but for couples from abroad their own insurance may not always cover all the costs, making it more expensive for them.

Though the sample for which follow-up was available was small, there was evidence that couples may change their minds and that opting for PGD to fulfill a child wish was not always an irrevocable choice. This finding implies that the couples should be re-evaluated after each PGD cycle so that alternative options can be (re)discussed. The follow-up of these patients will not only allow the medical aspects of the PGD cycle to be discussed (hormonal stimulation, number of embryos,...), but also it will provide an opportunity to investigate whether PGD is still the best treatment for a particular couple.

\section{References}

1 Handyside A, Kontogianni E, Hardy K, Winston R: Pregnancies from biopsied human preimplantation embryos sexed by Y-specific DNA amplification. Nature 1990; 344: 768-770.

2 Handyside AH, Lesko JG, Tarin JJ, Winston RM, Hughes MR: Birth of a normal girl after in vitro fertilization and preimplantation diagnostic testing for cystic fibrosis. N Engl J Med 1992; 327: 905-909.

3 Liu J, Lissens W, Silber S, Devroey P, Liebaers I, Van Steirteghem A: Birth after preimplantation diagnosis of the cystic fibrosis delta F508 mutation by polymerase chain reaction in human embryos resulting from intracytoplasmic sperm injection with epididymal sperm. JAMA 1994; 272: 1858-1860.

4 Goossens V, Sermon K, Lissens W et al: Clinical application of preimplantation genetic diagnosis for cystic fibrosis. Prenat Diagn 2000; 20: 571-581.

5 Goossens V, Sermon K, Lissens W et al: Improving clinical preimplantation genetic diagnosis for cystic fibrosis by duplex PCR using two polymorphic markers or one polymorphic marker in combination with the detection of the $\Delta$ F508 mutation. Mol Hum Reprod 2003; 9: 559-567.

6 Wells D: Advances in preimplantation genetic diagnosis. Eur J Obstet Gynecol Reprod Biol 2004; 115S: S97-S101.

7 Moutou C, Gardes N, Viville N: Duplex, triplex and quadruplex PCR for the preimplantation genetic diagnosis (PGD) of cystic fibrosis (CF), an exhaustive approach. Prenat Diagn 2004; 24 : 562-569.

8 Vandervorst M, Liebaers I, Sermon K et al: Successful preimplantation genetic diagnosis is related to the number of available cumulus-oocyte complexes. Hum Reprod 1998; 13: 3169-3176.

9 Devroey P, Van Steirteghem A: A review of ten years experience of ICSI. Hum Reprod Update 2004; 10: 19-28.

10 De Vos A, Van Steirteghem A: Aspects of biopsy procedures prior to preimplantation genetic diagnosis. Prenat Diagn 2001; 21: $767-780$.

11 Van Landuyt L, De Vos A, Joris $\mathrm{H}$ et al: Blastocyst formation in in vitro fertilization versus intracytoplasmic sperm injection cycles, influence of the fertilization procedure. Fertil Steril 2005; 83: $1397-1403$

12 Vandervorst $M$, Staessen C, Sermon $\mathrm{K}$ et al: The Brussels' experience of more than 5 years of clinical preimplantation genetic diagnosis. Hum Reprod Update 2000; 6: 364-373.

13 Bonduelle M, Liebaers I, Deketelaere V et al: Neonatal data on a cohort of 2889 infants born after ICSI (1991-1999) and of 2995 infants born after IVF (1983-1999). Hum Reprod 2002; 17: 671-694.

14 Heurckmans N, Vanhorenbeeck M, Vanderfaeillie A: Psychological aspects of preimplantation genetic diagnosis. Hum Reprod 1999; 14: 132

15 ESHRE PGD Consortium steering Committee: ESHRE Preimplantation Genetic Diagnosis (PGD) Consortium: data collection II (May 2000). Hum Reprod Update 2000; 15: 2673-2683.

16 Van der Ven $\mathrm{K}$, Meser L, Van der Ven $\mathrm{H}$ et al: Cystic fibrosis mutation screening in healthy men with reduced sperm quality. Hum Reprod 1996; 11: 513-517.

17 Foresta C, Ferlin A, Gianoroli L, Dallapiccola B: Guidelines for the appropriate use of genetic tests in infertile couples. Eur J Hum Genet 2002; 10: 303-312. 
18 Chillon M, Casals T, Mercier B et al: Mutations in the cystic fibrosis gene in patients with congenital absence of the vas deferens. N Engl J Med 1995; 22: 1475-1480.

19 Hilmon BC, Aitken ML, Constantinescu M: Pregnancy in patients with cystic fibrosis. Clin Obstet Gynecol 1996; 39: 70-86.

20 Sermon K, Van Steirteghem A, Liebaers I: Preimplantation genetic diagnosis. Lancet 2004; 363: 1633-1641.

21 ESHRE PGD Consortium Steering Committee: ESHRE Preimplantation Genetic Diagnosis Consortium: data collection III (May 2001). Hum Reprod 2002; 17: 233-246.

22 Bonduelle M, Van Assche E, Joris $\mathrm{H}$ et al: Prenatal testing in ICSI pregnancies: incidence of chromosomal anomalies in 1586 karyotypes and relation to sperm parameters. Hum Reprod 2002; 17: $2600-2614$

23 Anon: Report of the 11th annual meeting of International Working Group on Preimplantation Genetics: preimplantation genetic diagnosis - experience of 3000 clinical cycles. Reprod BioMed Online 2001; 3: 49-53.

24 Gerris JM: Single embryo transfer and IVF/ICSI outcome: a balanced appraisal. Hum Reprod Update 2005; 11: 105-121.

25 Harper J, Boelaert K, Geraedts J et al: ESHRE PGD Consortium data collection V: cycles from January to December 2002 with pregnancy follow-up to October 2003. Hum Reprod 2006; 21: $3-21$. 